\title{
Observation of Gas-Phase Molecular Dications Formed from Neutral Organics in Solution via Chemical Electron-Transfer Reactions by Using Electrospray Ionization Mass Spectrometry
}

\author{
Gary J. Van Berkel, Keiji G. Asano, and Scott A. McLuckey \\ Chemical and Analytical Sciences Division, Oak Ridge National Laboratory, Oak Ridge, Tennessee, USA
}

\begin{abstract}
The first observation of organic dications formed by multiple electron loss in electrospray mass spectra is reported. The dications of $\beta$-carotene, canthaxanthine, cobalt(II) octaethylporphyrin, and nickel(II) octaethylporphyrin were created in solution via chemical electrontransfer reactions and detected in the gas phase by electrospray ionization mass spectrometry (ES-MS) using a flow-injection experiment. The analytes were injected into a flowing solvent-oxidant stream $(10 \mu \mathrm{L} / \mathrm{min})$ composed of dried methylene chloride containing $\sim 0.1 \%$ by volume trifluoroacetic acid and $0.1 \%$ by volume antimony pentafluoride $\left(\mathrm{SbF}_{5}\right)$. The dications created in this oxidizing solvent system were preserved for detection by rapidly transferring them from the reactive solvent-oxidant system to the gas phase, where, in the absence of the solvent system, they were "long-lived" and amenable to mass analysis. This work demonstrates means to produce ions novel to ES-MS and means to detect and study by ES-MS species that are short-lived in solution. In addition, this work shows that electrospray ionization can potentially be used to generate gas-phase dications for mass spectrometric study that are difficult to produce directly from gas-phase neutrals by other ionization techniques (e.g., $\mathrm{M}^{2+}$ from $\beta$-carotene). (J Am Soc Mass Spectrom 1994, 5, 689-692)
\end{abstract}

I $n$ recent reports [1-5], we have shown that molecular radical cations, $\mathrm{M}^{+}$, can be formed in solution by electrochemical and chemical means, and can subsequently be transported to and detected in the gas phase using electrospray ionization mass spectrometry (ES-MS) [6-10]. The use of chemical electron-transfer reactions was demonstrated to be a sensitive and selective means to ionize in solution highly conjugated, aromatic, and heteroaromatic compounds for analysis by ES-MS. In this communication we report the first observation with ES-MS of gas-phase molecular dications of organics, that is, $\mathrm{M}^{2}$, that were formed in solution via chemical electron-transfer reactions [11].

Radical cations or other ionic species formed by gain or loss of electrons are not normally observed in electrospray (ES) mass spectra because the ES process does not normally [3] ionize the analyte directly. The nature of the ES ionization process, though not fully understood, is such that production of gas-phase analyte ions requires the presence of analyte ions in solution [11-15]. The 3-5-kV potential applied to the ana-

Address reprint requests to Dr. Gary J. Van Berkel, Chemical and Analytical Sciences Division, Oak Ridge National Laboratory, P. $O$. Box 2008/Bldg. 5510, Oak Ridge, TN 37831-6365.

lyte solution, which is responsible for the electrospray, merely assists the transfer of ionic analyte species already present in solution into the gas phase. As such, the types of ions normally observed in ES mass spectra are the cations or anions of salts and pseudomolecular species, for example, $[\mathrm{M}+\mathrm{nH}]^{\mathrm{n}+},[\mathrm{M}-\mathrm{nH}]^{\mathrm{n}-}$, and $[\mathrm{M}+\mathrm{nNa}]^{\mathrm{n}+}$, that are formed from species that can be ionized in solution via Brønsted or Lewis acid-base chemistry. These types of ionic species are stable in solution, that is, they have long solution lifetimes. Typically, radical cations or dications are much more reactive in solution, that is, they have short solution lifetimes.

We found that the dications of $\beta$-carotene, canthaxanthin, cobalt(II) octaethylporphyrin (CO(II)(OEP), and nickel(II) octaethylporphyrin could be created in solution and detected in the gas phase by ES-MS by using a flow-injection system with an appropriate oxidizing solvent stream. Such a system minimizes the time between creation of the ions in solution and the time at which they are transferred to the gas phase by means of the ES process. For the purposes of this communication, the discussion will focus on the $\beta$-carotene results. A detailed discussion of the results obtained with the other compounds can be found elsewhere [16]. 


\section{Experimental}

Electrospray experiments were carried out usirg a modified version of a Finnigan-MAT (San Jose, CA) ion trap mass spectrometer (ITMS) adapted to sample from ambient air [2, 3]. For continuous infusion, a syringe pump (Harvard Apparatus, Inc., Cambridge, MA) was used to pump analyte solutions at a rate of $10 \mu \mathrm{L} / \mathrm{min}$ through a short length of $500-\mu \mathrm{m}-\mathrm{i} . \mathrm{d}$. Teflon $^{\mathrm{B}}$ tubing (Upchurch Scientific, Oak Harbor, WA) that connected via a $1 / 16$ - to $1 / 32$-in. zero dead volume bulkhead reducing union (Valco, Houston, TX) and standard fittings to a $120-\mu \mathrm{m}-\mathrm{i} . \mathrm{d}$. (500- $\mu \mathrm{m}-\mathrm{o} . \mathrm{d}$.) dome-tipped needle within a pneumatically assisted ES source. Hlow-injection experiments were carried out at $10 \mu \mathrm{L} / \mathrm{min}$ by using the syringe pump to deliver the solvent-oxidant mixture at a constant rate, through Teflon tubing, to a Rheodyne (Cotati, CA) Model 7520 injector with a $0.5-\mu \mathrm{L}$ internal sample chamber and then through a short length $(\sim 15 \mathrm{~cm})$ of $100-\mu \mathrm{m}$-i.d. silica capillary to which the pneumatically assisted ES source was connected. The analyte, dissolved in methylene chloride, was injected into the flowing stream so that reaction with the oxidant occurred in the silica capillary just prior to the ES needle.

Methylene chloride was dried prior to use by elution through a bed of activated alumina and stored over alumina in an airtight flask. All analyte solutions were prepared via serial dilution with dry methylene chloride from a $1000-\mu \mathrm{M}$ stock solution of the respective analyte prepared in dry methylene chloride.

A $10-\mu \mathrm{M}$ solution of $\beta$-carotene in methylene chloride $/ 0.1 \%$ trifluoroacetic acid (TFA) $(\mathrm{v} / \mathrm{v})$ was prepared by first adding $10 \mu \mathrm{L}$ of TFA to $0.1 \mathrm{~mL}$ of a $1000-\mu \mathrm{M}$ solution of $\beta$-carotene in methylene chloride. This analyte-oxidant mixture was then diluted with methylene chloride to give the final solution composition. A $10-\mu \mathrm{M}$ solution of $\beta$-carotene in methylene chloride $/ 0.1 \%$ TFA $/ 0.1 \% \mathrm{SbF}_{5}(\mathrm{v} / \mathrm{v} / \mathrm{v})$ was prepared by first diluting an aliquot of the stock solution to 10 $\mu \mathrm{M}$ and then adding the TFA and $\mathrm{SbF}_{5}$ to the solution. A $10-\mu \mathrm{M}$ solution of $\beta$-carotene in methylene chloride $/ 0.1 \%$ TFA $(v / v)$ containing 2,3-dichloro-5,6dicyano-1,4-benzoquinone (DDQ; $100 \mu \mathrm{M}$ ) was prepared by diluting an aliquot of the $\beta$-carotene stock solution with methylene chloride and then adding the appropriate amount of TFA and a small volume of a concentrated stock solution of DDQ. The color of the analyte-oxidant solutions was monitored about every 15 min, both visually and by UV/visible spectrophotometry (Shimadzu UV/vis Model UV-2101, Kyoto, Japan) over the wavelength range of $200-800 \mathrm{~nm}$.

To acquire the tandem mass spectrum of the $\beta$ carotene dication, ion trap parameters were set such that only a small window of ions surrounding $\mathrm{m} / \mathrm{z}$ 268 that might be formed in a flow-injection experiment would be stored in the trap upon ion injection [17]. The trapped ions at $m / z 268$ were then collisionally dissociated mass-selectively by using the normal
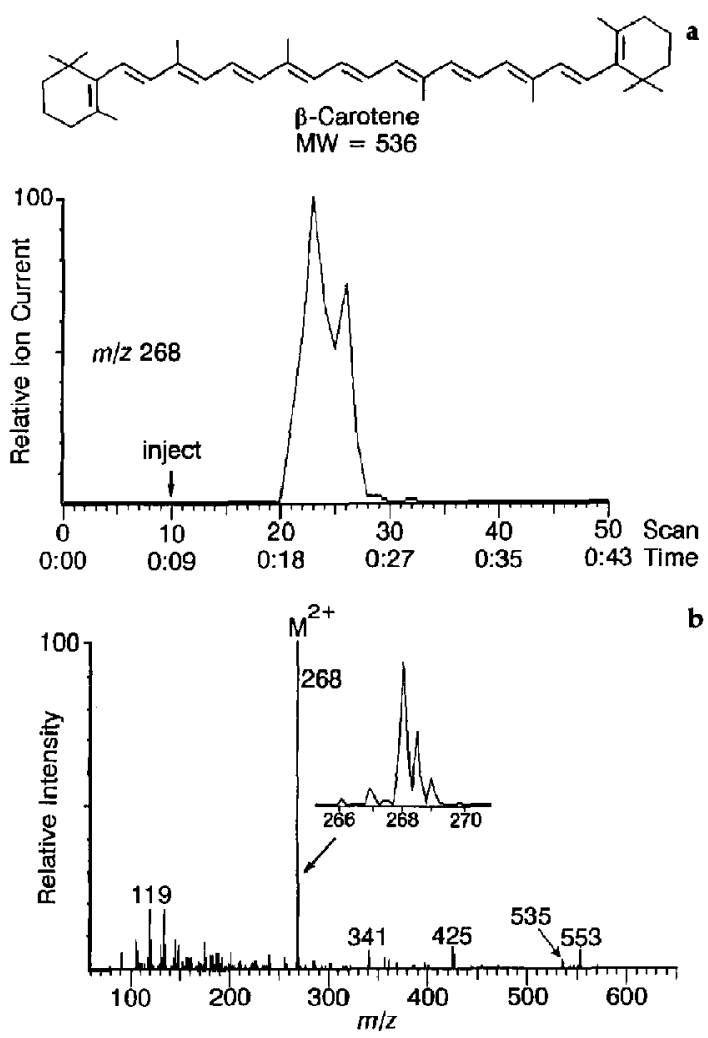

Figure 1. (a) Extracted ion current profile for $m / z 268$ obtained from the injection of $5 \mathrm{pmol}(0.5 \mu \mathrm{L})$ of $\beta$-carotene $(\mathrm{MW}=536$, dissolved in methylene chloride) into a flowing stream (10 $\mu \mathrm{L} / \min )$ composed of methylene $/ 0.1 \% \mathrm{TFA} / 0.1 \% \mathrm{SbF}_{5}(\mathrm{v} / \mathrm{v} / \mathrm{v})$. (b) The background-subtracted averaged mass spectrum obtained over the peak in the extracted ion current profile in (a). The inset in (b) shows the half-mass spacings of the isotope peaks around $m / z 268$ indicative of a doubly charged ion.

ion trap single-frequency resonance excitation mode of collisional activation [18].

\section{Results and Discussion}

Shown in Figure 1 are the extracted ion current profile for the $\beta$-carotene dication $(m / z 268)$ and the background-subtracted average mass spectrum obtained from a flow-injection experiment in which 5 pmol of $\beta$-carotene were injected into a flowing stream (10 $\mu \mathrm{L} / \mathrm{min}$ ) of methylene chloride containing about $0.1 \%$ by volume TFA and $0.1 \%$ by volume antimony pentafluoride $\left(\mathrm{SbF}_{5}\right)$. The major peak in the ES mass spectrum $(m / z 268)$ appears at the mass-to-charge ratio value expected for the molecular dication of $\beta$ carotene formed via an oxidation reaction involving the net loss of two electrons. Other ions of lesser abundance occur at low mass (e.g, $m / z$ 119) and may be fragment ions formed upon ion injection into the trap [3]. The higher mass ions at $m / z 535$ and 553 are 
probably also $\beta$-carotene reaction products, nominally $[\mathrm{M}-1]^{+}$and $[\mathrm{M}+17]^{+}$(probably $[\mathrm{M}+\mathrm{OH}]^{+}$).

The ion at $m / z 268$ was confirmed to be that of the dication, rather than a singly charged ion at one-half the molecular mass, by two pieces of data. First, as the insert in the spectrum in Figure $1 \mathrm{~b}$ shows, the isotope peaks for this ion, as observed in real time by using peak-profile mode, occur at half-mass intervals as expected for a doubly charged ion. The second piece of data stems from the tandem mass spectrum of $\mathrm{m} / \mathrm{z}$ 268 shown in Figure 2. The tandem mass spectrum contains product ions both at mass-to-charge ratio values less than and greater than that of the parent ion. Such a tandem mass spectrum is only possible if the parent ion is multiply charged. Ions in the tandem mass spectrum at values greater than $\mathrm{m} / \mathrm{z} 268$ are produced by charge-separation dissociations [18] that most likely occur along the polyene backbone of $\beta$ carotene. Such fragmentation normally results in pairs of singly charged complementary ions. Several such pairs of ions can be identified from among the ions observed in the tandem mass spectrum (e.g., $m / z$ $322 / 214,335 / 201$, and 348/188). Other ions observed in the tandem mass spectrum probably result from sequential fragmentation of these charge-separation products or from fragmentation of the parent ion with charge retention (i.e., doubly charged fragments).

The use of a flow-injection system is necessary for observation of the dications because of their limited lifetime in solution. We found that mixing $\beta$-carotene
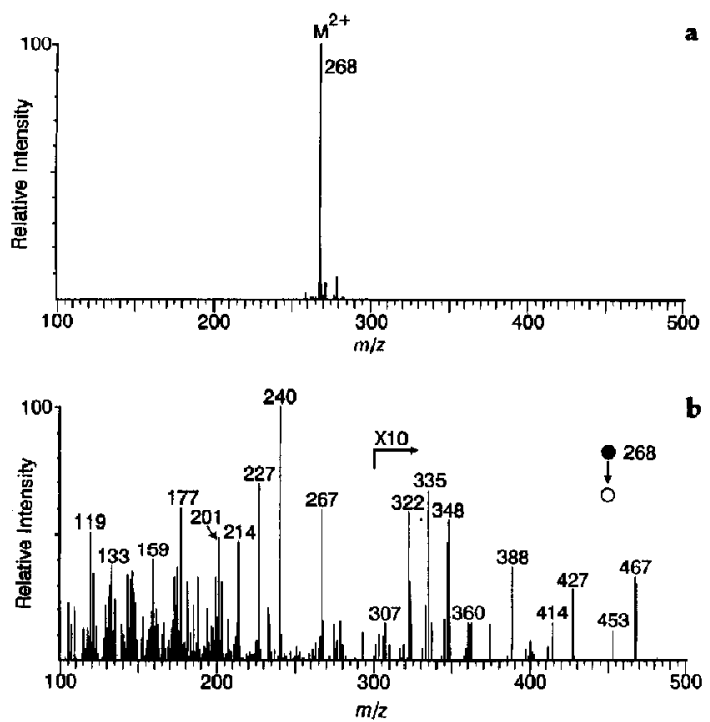

Figure 2. Tandem mass spertrum of $m / z .268$ ohserved in a flow-injection experiment carried out in the same manner as described in Figure 1. (a) ES mass spectrum acquired during a flow-injection experiment with instrumental parameters set to isolate a small window of ions surrounding $m / z \quad 268$. The single-frequency resonant excitation frequency is turned off. (b) Tandem mass spectrum of isolated ions at $m / z 268$ acquired by using the same instrumental parameters as in (a), but with the single-frequency resonant excitation frequency turned on. with TFA, DDQ, or $\mathrm{SbF}_{5}$ in an attempt to ionize the analyte was accompanied by a dramatic solution color change indicative of a chemical reaction (see Experimental section for details). Often solution color continued to change following mixing, indicating a series of reactions were occurring in solution. This was confirmed by changes in the ES mass spectra of these analyte-oxidant solutions with time. At $\sim 1 \mathrm{~min}$ following analyte-oxidant mixing, the ES mass spectra of the $\beta$-carotene solutions containing DDQ or $\mathrm{SbF}_{5}$ showed little evidence of ions that could be directly related to the analyte, and the ions that did appear continued to change with time. However, the ES mass spectrum obtained by continuous infusion of a methylene chloride $/ 0.1 \%$ TFA ( $/ \mathrm{v}$ ) solution of $\beta$-carotene was constant over several hours as was the color of the solution (spectrum not shown). The major ion observed in the high mass region of this spectrum appeared at $m / z 535$, which corresponds in mass to $[\mathrm{M}-1]^{+}$.

The observation of no identifiable $\beta$-carotene moıscular ionic species or observation of $[\mathrm{M}-1]^{+}$as the molecular species in these three continuous infusion experiments, rather than the dication observed in the flow-injection experiment, can be rationalized based on studies of the electrochemical oxidation of $\beta$-carotene by Kispert and co-workers [19-23]. They cited evidence from electrochemical, optical, and electron paramagnetic resonance work indicating facile electrochemical formation of $\beta$-carotene dications in methylene chloride followed by rapid proton loss to form $\left[\mathrm{M}^{2+}-\mathrm{H}^{+}\right]^{+}$. Therefore, the ion we observe at $m / z$ 535 probably is $\left[\mathrm{M}^{2+}-\mathrm{H}^{+}\right]^{+}$, formed by a solution reaction of the initially formed dication. Neither the dications nor $\left[\mathrm{M}^{2+} \cdot \mathrm{H}^{+}\right]^{+}$, if formed, are apparently stable in the solvent-oxidant systems containing DDQ or $\mathrm{SbF}_{5}$ for much longer than the 1-2 min that elapsed between analyte-oxidant mixing and the initial ES-MS analysis.

The yield of gaseous dications formed via the net two-electron oxidation in solution relies upon both the extent of the oxidation reaction and upon the reactivity (i.e., the stability or lifetime) of the dications in solution. The data presented here and the data of Kispert and co-workers [19-23] demonstrate that the dications of $\beta$-carotene are short-lived species in solution (lifetimes of a few tens of seconds at most). Under our experimental conditions, the analyte remained in contact with the oxidizing solvent system from the time of injection until complete peak elution for only 10-20 s (see Figure 1a). The dications formed by the oxidation reaction were preserved for detection by rapidly transferring them from solution to the gas phase, where, in the absence of solvent, they are "long-lived."

\section{Summary}

In this communication, we have reported chemical means to prepare dications from carotenoids and 
metalloporphyrins in solution and have shown by using the results for $\beta$-carotene that these ions can be transported to the gas phase via ES and mass analyzed. These dications formed by multiple electron loss are novel in ES mass spectra. However, it is expected that other aromatic, heteroaromatic, and highly conjugated systems (i.e., easy to oxidize compounds) might form multiply charged ions in solution via such electron-transfer reactions that can be successfully transported to the gas phase via ES. One potential analytical use of this technique is the study of dications that are difficult to produce directly in the gas phase via other ionization methods. The dication abundance in the electron ionization mass spectrum of $\beta$-carotene, for example, is only a few percent of the base peak. With the ES-MS technique described in this article, the dication is the base peak in the spectrum. In addition, because of the importance of carotenoids as a compound class and the general difficulty in analyzing carotenoids by some other mass spectrometry methods $[22,23]$, this ES-MS method of analysis may prove analytically useful for this compound class. More generally, the results presented here suggest that ES-MS might be used to detect and study ionic species formed by chemical means and by other means (e.g., electrochemically or photochemically) that are relatively short-lived in solution.

\section{Acknowledgment}

This research was sponsored by the United States Department of Energy, Office of Basic Energy Sciences, under contract DE-AC058401R21400 with Martin Marietla Energy Systems, Inc.

\section{References}

1. Van Berkel, G. J.; McLuckey, S. A.; Glish, G. L. Procedings of the 39th ASMS Conference on Mass Spectrometry and Allied Topics; Nashville, TN, May 1991; $\Lambda$ SMS: East Lansing, MI; PP $1237-1238$.
2. Van Berkel, G. J.; McLuckey, S. A.; Glish, G. L. Anal. Chem. 1991, 63, 2064-2068.

3. Van Berkel, G. J.; McLuckey, S. A.; Glish, G. L. Aral. Chem. 1992, 64, 1584-1593.

4. Asano, K. G.; Van Berkel, G. J. Proceedings of the 41st ASMS Conference on Mass Spectrometry and Alited Topics; San Francisco, CA, May 30-June 4, 1993, ASMS: Santa Fe, NM; pp $1068 a-1068 b$.

5. Van Berkel, G. J.; Asano, K. G. Anal. Chem., submitted.

6. Fenn, J. B.; Mann, M.; Meng, C. K.; Wong, S. K.; Whitehouse, C. M. Science 1989, 246, 64-71.

7. Fenn, J. B.; Mann, M.; Meng, C. K.; Wong, S. K.; Whitehouse, C. M. Mass Spectrom. Rev. 1990, 9, 37-71.

8. Smith, R. D.; Loo, J. A.; Edmonds, C. G.; Barinaga, C. J.; Udseth, H. R. Anal. Chem. 1990, 62, 882-899.

9. Huang, E. C.; Wachs, I.; Conboy, J. J.; Henion, J. D. Anal. Chem. 1990, 62, 713A-725A

10. Mann, M. Org. Mass Spectrom. 1990, 25, 575-587.

11. Ikonomou, M. G.; Blades, A. T.; Kebarle, P. Anul. Chem. 1991 , 63, 1989-1998.

12. Fenn, J. B. J. Am. Soc. Mass Spectrom. 1993, 4, 524-535.

13. Ashton, D. S.; Beddell, C. R.; Cooper, D. J.; Green, B. N.; Oliver, R. W. A. Org. Mass Spectrom. 1993, 28, 721-728.

14. Guevremont, R.; Siu, K.W. M.; Le Blanc, J. C. Y.; Berman, S. S. I. Am. Soc. Mass Spectrom. 1992, 3, 216-224.

15. Cheng, Z. L.; Siu, K. W. M.; Guevremont, R.; Berman, S. S. J. Am. Soc. Mass Spectrom. 1992, 3, 281-288.

16. Van Berkel, G. J.; Zhou, F. Anal. Chem., in press.

17. McLuckey, S. A.; Goeringer, D. E.; Glish, G. L. I. Am. Soc. Mass Spectrom. 1991, 2, 11-21.

18. Busch, K. L.; Glish, G. L.; McLuckey, S. A. Mass Spectrometry/ Mass Spectrometry: Techniques and Applications of Tandem Mass Spectrometry; VCH Publishers: New York, 1988.

19. Grant, J. L.; Kramer, V. J.; Ding, R.; Kispert, L. D. I. Am. Chem. Soc. 1988, 170, 2151-2157.

20. Khaled, M.; Hadjipetrou, A.; Kispert, L. D. J. Phys. Chem. 1990, 94, 5164-5169.

21. Khaled, M.; Hadjipetrou, A.; Kispert, L. D.; Allendoefer, R. D. J. Phys. Chem. 1991, 95, 2438-2442.

22. van Breemen, R. B.; Schmitz, H. H; Schwartz, S. J. Anal. Chem. 1993, 65, 965-969.

23. Takaichi, S. Org. Mass Spectrom. 1993, 28, 785-788. 